\title{
NÍVEIS DE FÓSFORO PARA CAPRINOS: PERDA ENDÓGENA FECALE EXIGÊNCIA LÍQUIDA PARA MANTENÇA ${ }^{1}$
}

\author{
MAURO SARTORI BUENO² e DORINHA MIRIAM SILBER SCHMIDT VITTI ${ }^{3}$
}

\begin{abstract}
RESUMO - Foram utilizados, no experimento, nove caprinos machos castrados, da raça Alpina, em dois períodos. Os animais permaneceram 28 dias em dieta experimental composta de feno e concentrado, sem suplementação de P, ou com $1 \mathrm{~g}$ ou $2 \mathrm{~g}$ de $\mathrm{P}$, na forma de fosfato bicálcico. No 21ํำ dia os animais receberam injeção de 7,4 MBq de ${ }^{32} \mathrm{P}$ na jugular, e coletaram-se amostras de sangue, fezes e urina, por sete dias, com o objetivo de avaliar o metabolismo do P. O aumento do consumo de P levou a aumento linear significativo do $\mathrm{P}$ excretado nas fezes, do $\mathrm{P}$ absorvido, do $\mathrm{P}$ endógeno fecal, da eficiência de absorção, e dos teores de $\mathrm{P}$ nas fezes e no plasma. $\mathrm{O}$ aumento do $\mathrm{P}$ endógeno fecal (Y) em função do consumo de $\mathrm{P}(\mathrm{X})$ pode ser descrito pela equação $\mathrm{Y}=10,36+0,58 \mathrm{X}(\mathrm{r}=0,94)$; a perda endógena mínima desses animais foi de $10,36 \mathrm{mg} / \mathrm{kg}$ de peso vivo/dia. A eficiência de absorção média foi de $65,76 \%$ para os animais suplementados. $\mathrm{O}$ teor de $\mathrm{P}$ na saliva não apresentou relação linear significativa com o $\mathrm{P}$ consumido, e não houve excreção de P pela urina.
\end{abstract}

Termos para indexação: fezes, fósforo radioativo, plasma, saliva, urina.

\author{
PHOSPHORUS LEVELS FOR GOATS: \\ ENDOGENOUS FECAL LOSS AND NET REQUIREMENT FOR MAINTENANCE
}

\begin{abstract}
Nine male castrated Alpine goats were used and the experiment was repeated twice. Animals were fed a diet consisted of hay and concentrate mixture without $\mathrm{P}$ supplementation, or with different amounts of dicalcium phosphate to give $1 \mathrm{~g}$ or $2 \mathrm{~g}$ P/animal/day for 28 days. At the $21^{\text {st }}$ day, 7,4 $\mathrm{MBq}^{32} \mathrm{P}$ were injected through jugular vein into each animal; and feces, blood and urine samples were collected during seven days to determine the $\mathrm{P}$ metabolism. Total fecal $\mathrm{P}$, absorbed $\mathrm{P}$, endogenous fecal $\mathrm{P}$, efficiency of absorption, feces and plasma $\mathrm{P}$ levels were positively related to $\mathrm{P}$ intake. The relationship between $\mathrm{P}$ intake $(\mathrm{X})$ and endogenous fecal $\mathrm{P}(\mathrm{Y})$ is described by the equation: $\mathrm{Y}=10.36+0.58 \mathrm{X}$ $(\mathrm{r}=0,94)$. The minimum endogenous $\mathrm{P}$ was $10,36 \mathrm{mg} / \mathrm{kg}$ live weight/day and efficiency of absorption was $65,76 \%$ for the supplemented treatments. Saliva P level was not related to P intake and there was not $\mathrm{P}$ excretion in the urine.
\end{abstract}

Index terms: feces, radioactive phosphorus, plasma, urine, saliva.

\section{INTRODUÇÃO}

O estudo da utilização do P na espécie caprina é necessário para estimar a quantidade exata do elemento a ser fornecida aos animais diariamente, pois

\footnotetext{
${ }^{1}$ Aceito para publicação em 6 de novembro de 1998. Financiado pela FAPESP.

${ }^{2}$ Zoot. Dr., Instituto de Zootecnia, Caixa Postal 60, CEP 13460-000 Nova Odessa, SP. Email: msbueno@izsp.br

${ }^{3}$ Bióloga, Dra., CENA/USP, Piracicaba, SP.
}

o excesso do elemento pode causar urolitíase em machos, e o fornecimento insuficiente pode levar a distúrbios reprodutivos e diminuição da produção.

Os requerimentos nutricionais da espécie caprina são menos conhecidos que os das demais espécies de ruminantes domésticos. O National Research Council (1981) não menciona a perda endógena mínima nem a eficiência de absorção de $\mathrm{P}$, e os dados referentes a este elemento são extrapolados das espécies ovina e bovina. 
A absorção de $\mathrm{P}$ em ruminantes é realizada por transporte passivo, em direta relação ao consumo (Braithwaite, 1985) ou seja, quanto maior a concentração intestinal, maior a absorção, e o transporte ativo pode ser determinado por uma maior demanda pelo animal. A homeostase de $\mathrm{P}$ em ruminantes é conseguida através da secreção do $\mathrm{P}$ pela saliva (P endógeno) e excreção pelas fezes, de acordo com a demanda do animal (Scott et al., 1985). A excreção de P pela urina é muito pouca, ou seja: menos que 1\% (Thompson Junior, 1978), e pode se elevar quando a ingestão de $\mathrm{P}$ excede à demanda do animal e os valores plasmáticos ultrapassam o limiar renal (Field et al., 1984).

Nos ruminantes a determinação da perda endógena é importante, já que nesses animais a excreção do $\mathrm{P}$ endógeno pode exceder o $\mathrm{P}$ não digerido do alimento (Georgievskii, 1982), e assim, subestimar a absorção do elemento.

A estimativa da exigência nutricional dos macroelementos inorgânicos é realizada a partir do conhecimento da perda endógena mínima do animal em mantença. Esta pode ser estimada mediante experimento em que animais são submetidos a dietas com níveis variados do elemento, traçando-se uma curva de regressão para o nível zero de ingestão (Agricultural Research Council, 1980).

A determinação do $\mathrm{P}$ endógeno fecal envolve o uso do radionuclídeo ${ }^{32} \mathrm{P}$ como traçador, e os princípios básicos do método foram descritos por Lofgreen et al. (1952), Luick \& Lofgreen (1957) e Vitti (1989).

$\mathrm{O}$ conhecimento da eficiência de absorção do $\mathrm{P}$ da dieta pelos animais e fatores que a regulam são necessários para se transformar a exigência líquida em exigência dietética diária, utilizados em tabelas de exigências nutricionais dos animais domésticos. Assim, valores de eficiência de absorção superestimados levariam a um fornecimento insuficiente do elemento para determinada categoria animal, acarretando diminuição de produção, e valores subestimados poderiam levar ao fornecimento excessivo, com prejuízos econômicos e para a saúde do animal.

O presente estudo teve como objetivo avaliar o metabolismo do $\mathrm{P}$ em caprinos alimentados com níveis crescentes do elemento na dieta.

\section{MATERIAL E MÉTODOS}

Foram utilizados nove caprinos machos castrados da raça Alpina, com, aproximadamente, um ano de idade, e peso vivo médio de $34,7 \mathrm{~kg}$, mantidos em gaiolas de digestibilidade com coletor de fezes e urina. Os animais foram alimentados com $700 \mathrm{~g} / \mathrm{dia}$ de feno de coast cross (Cynodon dactylon (L.) Pears) e $200 \mathrm{~g} /$ dia de concentrado e distribuídos em três tratamentos: sem suplementação, ou com um ou dois gramas de $\mathrm{P} /$ animal/dia, provenientes do fosfato bicálcico, durante 28 dias (Tabelas 1 e 2). No $21^{\circ}$ dia, pela manhã, retirou-se amostra de sangue, para determinação do teor de $\mathrm{P}$ inorgânico no plasma, e aplicouse injeção de 7,4 MBq de ${ }^{32} \mathrm{P}\left(\mathrm{Na}_{2} \mathrm{HPO}_{4}\right.$ - livre de carregador) na jugular esquerda de cada animal. Nos sete dias seguintes, pela manhã, foram coletadas amostras de sangue, fezes e urina. Após a coleta de dados, os animais permaneceram por mais sete dias nas gaiolas, para decaimento biológico e, então, foram alojados em baias

TABELA 1. Composição das dietas suplementadas com diferentes quantidades de $P$.

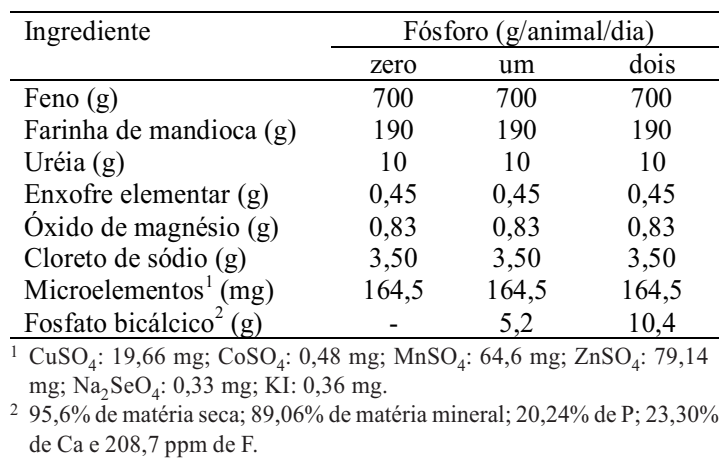

TABELA 2. Composição químico-bromatológica das dietas (em porcentagem da matéria seca) suplementadas com diferentes quantidades de $\mathbf{P}$.

\begin{tabular}{lrrr}
\hline Composição & \multicolumn{3}{c}{ Fósforo (g/animal/dia) } \\
\cline { 2 - 4 } & zero & \multicolumn{1}{c}{ um } & dois \\
\hline Matéria seca (\%) & 82,56 & 82,56 & 82,56 \\
Proteína bruta & 8,12 & 8,25 & 8,47 \\
Fibra bruta & 30,03 & 30,42 & 30,07 \\
Extrato etéreo & 1,33 & 1,34 & 1,31 \\
Extrativo-não-nitrogenado & 56,91 & 55,82 & 55,10 \\
Nutrientes digestíveis totais & 70,28 & 70,95 & 70,63 \\
Ca & 0,22 & 0,38 & 0,54 \\
P & 0,10 & 0,23 & 0,37 \\
Ca:P & $2,2: 1$ & $1,7: 1$ & $1,5: 1$ \\
\hline
\end{tabular}


coletivas, onde permaneceram por 45 dias. Posteriormente, foram alocados aleatoriamente nos tratamentos, e o procedimento anterior foi repetido.

As amostras de fezes foram pesadas diariamente, e retirou-se aliquota de $10 \%$, as quais foram armazenadas em congelador e, posteriormente, formaram uma amostra composta por animal. $\mathrm{O}$ teor de $\mathrm{P}$ das amostras dos alimentos, suplemento fosfórico, e fezes, foi determinado nas cinzas solubilizadas, pelo método colorimétrico, descrito por Sarruge \& Haag (1974), e a atividade, pelo efeito Cerenkov (International Atomic Energy Agency, 1979), em espectrômetro de cintilação líquida.

As amostras de sangue foram coletadas em tubos heparinizados, centrifugadas a $3.000 \mathrm{rpm}$, por $10 \mathrm{minu}-$ tos, e o plasma, coletado e desproteinizado com solução de ácido tricloroacético $(10 \%)$, para determinação do teor de P inorgânico (Fiske \& Subbarow, 1925) e da atividade, pelo efeito Cerenkov.

As amostras de urina foram medidas diariamente, e alíquotas de $10 \%$ foram retiradas e armazenadas em congelador, as quais formaram amostra composta por animal. O teor de $\mathrm{P}$ inorgânico das amostras de urina e saliva foram determinados como no plasma.

Através das atividades específicas (atividade/ $P$ inorgânico) do plasma e das fezes foram determinados o $\mathrm{P}$ endógeno e a eficiência de absorção do $\mathrm{P}$ das dietas como em Vitti (1989).

Foi utilizado um delineamento completamente casualizado, repetido em dois períodos (três tratamentos e dois períodos) com três repetições por tratamento por período; os dados foram submetidos à análise de regressão, e efetuaram-se correlações entre as variáveis estudadas.

\section{RESULTADOS E DISCUSSÃO}

A Tabela 3 mostra os dados de consumo e excreção do P, eficiência de absorção e balanço de P, assim como os teores de P no plasma e na saliva dos animais submetidos a níveis crescentes de $\mathrm{P}$ na dieta. Não foi detectada diferença significativa entre períodos $(\mathrm{P}>0,05)$ em nenhuna das variáveis estudadas.

A quantidade de $\mathrm{P}$ consumida aumentou consideravelmente com a suplementação crescente de fosfato bicálcico. Os animais sem suplementação fosfórica ingeriram pequena quantidade de $\mathrm{P}$ e isso foi devido aos baixos teores de $\mathrm{P}$ presentes na dieta (Tabela 2). O National Research Council (1981) preconiza o valor de 1,4 g de P/animal/dia para caprinos em mantença apenas e em condições de estabulação total e atividade física mínima. Desta maneira, as quantidades de $\mathrm{P}$ consumidas diariamente podem ser consideradas como insuficiente, adequada, e elevada, para os animais que não receberam suplementação ou receberam um ou dois g de $\mathrm{P} /$ dia, respectivamente.

A relação cálcio:fósforo dos tratamentos, apesar de mais elevada para os animais sem suplementação fosfórica, encontra-se dentro dos valores considerados normais para ruminantes (Salviano, 1996).

A quantidade diária de $\mathrm{P}$ excretado nas fezes $(\mathrm{Y})$ apresentou efeito linear positivo $(\mathrm{P}<0,01)$ com o $\mathrm{P}$ consumido $(X)$, expressa pela equação $\mathrm{Y}=19,67+$ 0,79X $(\mathrm{r}=0,89)$. O aumento da quantidade de $\mathrm{P}$ excretado nas fezes em função do aumento do $\mathrm{P}$ consumido também foi encontrado por Akinsoyinu (1986)

TABELA 3. Metabolismo do $P$ em caprinos alimentados com níveis crescentes do elemento na dieta (média \pm desvio-padrão).

\begin{tabular}{|c|c|c|c|c|}
\hline \multirow[t]{2}{*}{ Variável } & \multicolumn{3}{|c|}{ Fósforo (g/animal/dia) } & \multirow{2}{*}{$\begin{array}{c}\text { Regressão } \\
\text { linear }\end{array}$} \\
\hline & zero & $\mathrm{um}$ & dois & \\
\hline Peso vivo médio $(\mathrm{kg})$ & $34,4 \pm 1,1$ & $34,8 \pm 0,8$ & $34,8 \pm 0,9$ & - \\
\hline $\mathrm{P}$ consumido (mg/kg PV) & $22,43 \pm 4,60$ & $51,74 \pm 2,86$ & $81,49 \pm 6,03$ & - \\
\hline $\mathrm{P}$ excretado nas fezes $(\mathrm{mg} / \mathrm{kg} \mathrm{PV})$ & $34,60 \pm 2,86$ & $66,12 \pm 12,65$ & $80,93 \pm 13,72$ & $* *$ \\
\hline P endógeno fecal (mg/kg PV) & $20,91 \pm 4,19$ & $45,33 \pm 8,94$ & $55,71 \pm 6,27$ & $* *$ \\
\hline $\mathrm{P}$ absorvido (mg/kg PV) & $8,77 \pm 6,57$ & $30,95 \pm 10,68$ & $56,27 \pm 6,54$ & $* *$ \\
\hline Balanço de P (mg/kg PV) & $-12,17 \pm 3,12$ & $-14,38 \pm 13,48$ & $0,56 \pm 10,66$ & NS \\
\hline Eficiência de absorção (\%) & $43,24 \pm 17,51$ & $61,65 \pm 19,39$ & $69,86 \pm 8,75$ & $* *$ \\
\hline P inorgânico no plasma (mg/dL) & $4,86 \pm 1,11$ & $6,06 \pm 1,61$ & $6,27 \pm 0,81$ & $*$ \\
\hline$P$ saliva $(\mathrm{mg} / \mathrm{dL})$ & $80,20 \pm 16,82$ & $83,99 \pm 33,74$ & $89,39 \pm 27,67$ & NS \\
\hline$\% \mathrm{P}$ nas fezes & $0,13 \pm 0,014$ & $0,24 \pm 0,073$ & $0,31 \pm 0,042$ & $* *$ \\
\hline
\end{tabular}


em caprinos, e Braithwaite (1985) e Louvandini \& Vitti (1994) em ovinos.

A quantidade de $\mathrm{P}$ excretado nas fezes foi maior que o $\mathrm{P}$ consumido pelos animais sem suplementação e pelos que receberam $1 \mathrm{~g} /$ dia, e levemente inferior pelos que receberam $2 \mathrm{~g} /$ dia. Desta maneira, o balanço positivo de $\mathrm{P}$ só foi constatado em alguns animais que receberam a maior suplementação. Isso não significa que a homeostase só foi conseguida neste tratamento, pois sendo, a absorção do P, passiva (Braithwaite, 1984), o aumento da quantidade de $\mathrm{P}$ absorvido acima das necessidades levou a uma maior secreção salivar de $\mathrm{P}$ e maior perda fecal (Braithwaite, 1985; Scott et al., 1985), o que contribuiu para o aumento do $\mathrm{P}$ excretado nas fezes. A correlação positiva significativa $(\mathrm{P}<0,01)$ entre $\mathrm{P}$ absorvido e P excretado ( $\mathrm{r}=0,72)$ (Tabela 4$)$ corrobora esta afirmação.

O P endógeno fecal (Y) mostrou aumento linear $(\mathrm{P}<0,01)$, em função do $\mathrm{P}$ consumido $(\mathrm{X})$, representado pela equação $\mathrm{Y}=10,36+0,58 \mathrm{X}(\mathrm{r}=0,94)$. $\mathrm{O}$ aumento do $\mathrm{P}$ endógeno fecal causado pelo aumento da ingestão de $\mathrm{P}$, deve-se ao aumento da quantidade de $\mathrm{P}$ absorvido, que após ser secretado pela saliva é perdido em maior quantidade pelas fezes. Esse fato pode ser confirmado pela correlação positiva $(\mathrm{r}=0,84$, $\mathrm{P}<0,01)$ entre o $\mathrm{P}$ absorvido e o $\mathrm{P}$ endógeno fecal (Tabela 4). Essa tendência de aumento do P endógeno fecal concorda plenamente com os resultados de Challa \& Braithwaite (1988) e Challa et al. (1989) com bezerros em crescimento, Braithwaite (1984), Braithwaite (1985) e Louvandini \& Vitti (1994) com ovinos, e Akinsoyinu (1986), com caprinos.

Foi observada uma correlação positiva ( $\mathrm{r}=0,94$; $\mathrm{P}<0,01$ ) entre o $\mathrm{P}$ excretado e o $\mathrm{P}$ endógeno fecal, denotando que o aumento da excreção fecal de P é acompanhado de aumento de $\mathrm{P}$ endógeno fecal. A porcentagem de $\mathrm{P}$ endógeno no $\mathrm{P}$ total das fezes foi de 60,$5 ; 68,6$ e $68,8 \%$.

A regressão para o nível zero de $\mathrm{P}$ forneceu $\mathrm{o}$ valor de $10,36 \mathrm{mg} / \mathrm{kg}$ de peso vivo, que seria, segundo o Agricultural Research Council (1980), a perda endógena fecal mínima de $\mathrm{P}$, que somado à perda urinária, representaria a exigência líquida para mantença. Como não foi encontrada excreção urinária de P nesses animais, esse valor poderia ser considerado como a exigência líquida para mantença de caprinos nas condições experimentais.

$\mathrm{O}$ valor de perda endógena mínima de $\mathrm{P}$ encontrado concorda com o preconizado em ovinos (Agricultural Research Council, 1980; Braithwaite, 1984; Louvandini \& Vitti, 1994). O valor observado por Akinsoyinu (1986) em caprinos anões (15,6 $\mathrm{mg} / \mathrm{kg}$ de peso vivo/dia) foi superior ao deste estudo, e, provavelmente, deve-se à maior demanda pelo elemento por aqueles animais, e maior consumo de MS, visto estarem ganhando peso.

O National Research Council (1985) indicou o valor de $20 \mathrm{mg} / \mathrm{kg}$ de peso vivo como perda endógena mínima em ovinos, valor superior ao encontrado neste estudo, devido à maior excreção de $\mathrm{P}$ endógeno em animais em produção.

Braithwaite (1985) encontrou o valor de $23,8 \mathrm{mg} \mathrm{P} / \mathrm{kg}$ peso vivo como perda endógena fecal mínima em ovinos em crescimento com ganho de peso diário ao redor de $200 \mathrm{~g}$, concluindo que animais em produção apresentam maior perda endógena fecal que os animais em mantença.

Desta maneira, pode-se concluir que os animais devem absorver $10,36 \mathrm{mg} \mathrm{P} / \mathrm{kg}$ de peso vivo/dia para suprir as perdas inevitáveis. Portanto, os animais sem suplementação absorveram quantidade de $\mathrm{P}$ inferior às perdas inevitáveis, e esse fato provavelmente refletiu nos menores valores de $\mathrm{P}$ plasmáticos encontrados.

TABELA 4. Correlações entre algumas variáveis avaliadas.

\begin{tabular}{|c|c|c|c|c|c|c|c|}
\hline Variável & $\mathrm{P}$ consumido & P excretado & $\mathrm{P}$ endógeno & $\mathrm{P}$ absorvido & Eficiência de absorção & P no plasma & $\mathrm{P}$ na saliva \\
\hline P excretado & $0,89^{*}$ & & & & & & \\
\hline P endógeno & $0,90 * *$ & $0,94 * *$ & & & & & \\
\hline $\mathrm{P}$ absorvido & $0,94 * *$ & $0,72 * *$ & $0,84 * *$ & & & & \\
\hline Eficiência de absorção & $0,59 * *$ & $0,33^{\mathrm{NS}}$ & $0,60 * *$ & $0,80 * *$ & & & \\
\hline P no plasma & $0,56^{*}$ & $0,35^{\mathrm{NS}}$ & $0,55^{*}$ & $0,70 * *$ & $0,78 * *$ & & \\
\hline P na saliva & $0,23^{\mathrm{NS}}$ & $0,20^{\mathrm{NS}}$ & $0,17^{\mathrm{NS}}$ & $0,16^{\mathrm{NS}}$ & $0,10^{\mathrm{NS}}$ & $0,11^{\mathrm{NS}}$ & \\
\hline$\%$ P nas fezes & $0,84 * *$ & $0,96 * *$ & $0,91 * *$ & $0,65^{*}$ & $0,28^{\mathrm{NS}}$ & $0,40^{\mathrm{NS}}$ & $0,02^{\mathrm{NS}}$ \\
\hline
\end{tabular}


Para converter o valor de perda endógena mínima em requerimento para mantença, pode-se utilizar o valor de eficiência de absorção de $65,76 \%$, média dos tratamentos suplementados, obtendo-se o valor de $15,75 \mathrm{mg} / \mathrm{kg}$ peso vivo/dia como exigência dietética diária em caprinos em mantença. Este valor é menor que o utilizado pelo National Research Council (1981).

A quantidade de $\mathrm{P}$ absorvido (Y) pelos animais apresentou relação linear positiva $(\mathrm{P}<0,01)$, em função do $\mathrm{P}$ consumido $(\mathrm{X})$, representado pela equação $\mathrm{Y}=-10,11+0,80 \mathrm{X}(\mathrm{r}=0,94)$. O aumento da quantidade de $\mathrm{P}$ absorvido foi conseguido por maior ingestão de P, somado a aumento na eficiência de absorção do elemento, o que concorda com Braithwaite (1984), Challa \& Braithwaite (1988) e Louvandini \& Vitti (1994).

A eficiência de absorção do P (Y) das dietas pelos animais apresentou efeito linear positivo $(\mathrm{P}<0,05)$ com o consumo de $\mathrm{P}(\mathrm{X})$, sendo descrita pela equação $\mathrm{Y}=35,42+0,44 \mathrm{X}(\mathrm{r}=0,59)$. $\mathrm{O}$ aumento na eficiência de absorção encontrado concorda com Braithwaite (1985), denotando o efeito da suplementação com $\mathrm{P}$ proveniente do fosfato bicálcico, que parece possuir maior disponibilidade que o $\mathrm{P}$ da dieta básica. Challa et al. (1989), em estudo com bezerros em crescimento, também observaram menor eficiência de absorção em dietas sem suplementação de P na forma inorgânica, em que todo o P provinha do alimento. Os valores encontrados nos animais suplementados são similares aos citados por Kessler (1981) em relação a caprinos, e superiores aos valores de Vitti et al. (1991) relativos a ovinos suplementados com fosfato bicálcico.

Quando se subtraiu o efeito da eficiência de absorção do P da dieta (P orgânico), obtido pelos animais sem suplementação, foram encontrados os valores de $76,2 \%$ e 80,3\% em relação à eficiência de absorção do $\mathrm{P}$ do fosfato bicálcico para os animais com suplementação de $1 \mathrm{~g}$ e $2 \mathrm{~g}$ P/dia, respectivamente.

A regressão entre consumo de $\mathrm{P}$ e eficiência de absorção, apesar de significativa estatisticamente $(\mathrm{P}<0,01)$, apresentou valor $\mathrm{r}$ muito baixo $(0,59)$ e valores elevados de desvio-padrão, devido às grandes variações individuais. Pode-se, então, concordar com Field et al. (1984) e Solomon et al. (1991), que observaram a existência de grande variação na eficiência de absorção entre animais que receberam mesma quantidade de $\mathrm{P}$, ligada às suas características genéticas.

O P inorgânico no plasma (Y) apresentou efeito linear positivo e significativo $(\mathrm{P}<0,05)$ com o consumo do elemento $(\mathrm{X})$, descrito pela equação $\mathrm{Y}=4,32+$ 0,029 X ( $(\mathrm{r}=0,54)$, e está de acordo com Braithwaite (1985) e Scott et al. (1985), em estudo com ovinos, e Challa \& Braithwaite (1988) e Challa et al. (1989), com bezerros. As correlações positivas e significativas entre $\mathrm{P}$ absorvido e $\mathrm{P}$ inorgânico no plasma ( $\mathrm{r}=0,70$; $\mathrm{P}<0,01)$, e entre $\mathrm{P}$ endógeno fecal e $\mathrm{P}$ inorgânico no plasma $(\mathrm{r}=0,55 ; \mathrm{P}<0,05)$ (Tabela 4) denotam a estreita ligação entre essas variáveis.

O teor de P inorgânico no plasma encontrado nos animais sem suplementação fosfórica foi muito inferior aos valores dos tratamentos com suplementação. Tais resultados sugerem que a absorção de quantidade insuficiente de $\mathrm{P}$, ou seja, abaixo da perda endógena mínima, foi responsável por esse valor. Desta maneira, o valor de 4,0 $\mathrm{mg} / \mathrm{dL}$, preconizado pelo National Research Council (1985) como o limiar de carência para ovinos, parece ser inadequado para caprinos, e então será, para esta espécie, em torno de $5,0 \mathrm{mg} / \mathrm{dL}$. Quando os animais receberam suplementação de $\mathrm{P}$, a concentração plasmática foi superior a $6 \mathrm{mg} / \mathrm{dL}$, o que indica que estes valores refletem absorção acima da exigência de mantença.

Os teores de $\mathrm{P}$ inorgânico plasmático observados são inferiores aos encontrados por Akinsoyinu (1986) em caprinos anões com consumo elevado de P. Já Unanian \& Silva (1985) e Girdhar et al. (1991) citam, quanto a caprinos, amplitude de valores similares aos encontrados neste estudo.

A porcentagem de $\mathrm{P}$ nas fezes (Y) mostrou aumento $(\mathrm{P}<0,01)$ em função do $\mathrm{P}$ consumido $(\mathrm{X})$, e é descrita pela equação $\mathrm{Y}=0,073+0,0029 \mathrm{X}(\mathrm{r}=0,84)$. Esse resultado está de acordo com Vitti et al. (1988) e Sanson et al. (1990), que também encontraram relação entre o P consumido e o teor de $\mathrm{P}$ nas fezes de bovinos. Foram observadas correlações significativas entre a porcentagem de $\mathrm{P}$ nas fezes e o $\mathrm{P}$ excretado, o P endógeno e o P absorvido (Tabela 4), o que evidencia sua utilidade como indicador do "status" de P nesses animais. Assim, a porcentagem de $\mathrm{P}$ nas fezes pode ser utilizada como indicador de consumo e absorção de $\mathrm{P}$ na espécie caprina, o que 
está de acordo com Rosa (1988), que afirmou que esse parâmetro fornece a melhor indicação da ingestão desse elemento por ruminantes.

A concentração de $\mathrm{P}$ na saliva não apresentou efeito $(\mathrm{P}>0,05)$ com o $\mathrm{P}$ consumido ou absorvido, o que indica que a concentração de $P$ na saliva não foi um bom indicador do consumo ou absorção de $\mathrm{P}$, e desta maneira, concorda com Vitti et al. (1988) em estudo com bezerros. Parece que o método de coleta de saliva da boca do animal não representa a secreção salivar da parótida, que, segundo Ternouth et al. (1985), é responsável pela reciclagem do P endógeno.

\section{CONCLUSÕES}

1. O consumo de P determina a absorção, a excreção fecal total e endógena do elemento.

2. O balanço de $\mathrm{P}$ e sua concentração na saliva não são indicadores do consumo do elemento.

3. A porcentagem de $\mathrm{P}$ nas fezes reflete o consumo do elemento.

4. O teor de P no plasma é um bom indicador de consumo insuficiente de $\mathrm{P}$.

5. A exigência líquida de $P$ para mantença de caprinos nas condições experimentais é de $10,36 \mathrm{mg} / \mathrm{kg}$ de peso vivo/dia.

\section{REFERÊNCIAS}

AGRICULTURAL RESEARCH COUNCIL. The nutrient requirement of ruminant livestock. Slough: Commonwealth Agricultural Bureaux, 1980. $351 \mathrm{p}$.

AKINSOYINU, A.O. Minimum phosphorus requirements of the dwarf goat for maintenance. Tropical Agriculture, Trinidad, v.63, n.4, p.333-335, Oct. 1986.

BRAITHWAITE, G.D. Endogenous faecal loss of phosphorus in growing lambs and the calculation of phosphorus requirements. Journal of Agricultural Science, Cambridge, v.105, n.1, p.67-72, Aug. 1985.

BRAITHWAITE, G.D. Some observation on phosphorus homeostasis and requirements of sheep. Journal of Agricultural Science, Cambridge, v.102, n.2, p.295306, Apr. 1984.
CHALLA, J.; BRAITHWAITE, G.D. Phosphorus and calcium metabolism in growing calves with special emphasis on phosphorus homeostasis. 1. Studies of the effect of changes in the dietary phosphorus intake on phosphorus and calcium metabolism. Journal of Agricultural Science, Cambridge, v.110, n.3, p.573581, June 1988.

CHALLA, J.; BRAITHWAITE, G.D.; DHANOA, M.S. Phosphorus homeostasis in growing calves. Journal of Agriculture Science, Cambridge, v.112, n.2, p.217-226, Aug. 1989.

FIELD, A.C.; WOOLLIANS, J.A.; DINGWALL, R.A.; MUNRO, C.S. Animal and dietary variation in absorption and metabolism of phosphorus by sheep. Journal of Agricultural Science, Cambridge, v.103, n.2, p.283-291, Oct. 1984.

FISKE, C.H.; SUBBARROW, Y. The calorimetric determination of phosphorus. Journal of Biological Chemistry, Baltimore, v.66, n.2, p.375-400, Mar. 1925.

GEORGIEVSKII, V.I. The physiological role of macroelements. In: GEORGIEVISKII, V.I.; ANNENKOV, B.N.; SAMOKHIN, V.T. (Eds.). Mineral nutrition of animals. London: Butterworths, 1982. p.257-271.

GIRDHAR, N.; LALL, D.; PATHAK, N.N. Effect of Leucaena leucocephala as sole ration on nutrient utilisation, and body weight in goats. Journal of Agricultural Science, Cambridge, v.116, n.2, p.303307, Apr. 1991.

INTERNATIONAL ATOMIC ENERGY AGENCY. Laboratory training manual on the use of nuclear techniques in animal research. Vienna: IAEA, 1979. 299p. (Technical Report Series 193).

KESSLER, J. Eléments minéraux majeurs chez la chèvre:données de base et apports recommandés. In: MORAND-FEHR, P.; BOURBOUZE, A.; SIMIANE, M. (Eds.). Nutrition e System d'Alimentation de la Chèvre. Tours: INRA/ ITOVIC, 1981. p.196-209.

LOFGREEN, G.P.; KLEIBER, M.; SMITH, A.H. The excretion of injected ${ }^{32} \mathrm{P}$ into the gastrointestinal tract of young calf. Journal of Nutrition, Philadelphia, v.47, n.4, p.56-59, Apr. 1952.

LOUVANDINI, H.; VITTI, D.M.S.S. Perda endógena de fósforo em ovinos com diferentes níveis do elemento na dieta. Pesquisa Agropecuária Brasileira, Brasília, v.29, n.1, p.145-149, jan. 1994. 
LUICK, J.R.; LOFGREEN, G.P. An improved method for determination of metabolic fecal phosphorus. Journal of Animal Science, Champaign, v.16, n.1, p.201-206, Feb. 1957.

NATIONAL RESEARCH COUNCIL. (Washington, DC). Nutrient requirements of goat: Angora, dairy and meat goat in temperate and tropical countries. Washington, DC: National Academic Press, 1981. 87p.

NATIONAL RESEARCH COUNCIL. (Washington, DC). Nutrient requirements of sheep: Washington, DC: National Academic Press, 1985. 99p.

ROSA, I.V. Cálcio e fósforo na nutrição de ruminantes. In: VALVASORI, E. (Ed.). Encontro sobre nutrição mineral para ruminantes. Nova Odessa: Instituto de Zootecnia, 1988. p.46-63.

SALVIANO, L.M.C. Efeito de diferentes proporções de cálcio e fósforo sobre as perdas endógenas e absorção real de fósforo em ovinos. Piracicaba: USP-CENA, 1996. 84p. Tese de Doutorado.

SANSON, D.W.; WALKER, G.L.; CLANTON, D.C.; ESKRIDGE, K.M. Relationship between phosphorus intake and blood or fecal phosphorus in gestating cow. Journal of Range Management, Denver, v.43, n.3, p.238-241, May 1990.

SARRUGE, J.R.; HAAG, H.P. Análises químicas em plantas. Piracicaba: USP-ESALQ, 1974. 56p.

SCOTT, D.; WHITELAW, F.G.; BUCHAN, W.; BRUCE, L.A. The effect of variation in phosphorus intake on salivary phosphorus secretion, net intestinal phosphorus absorption and faecal endogenous phosphorus excretion in sheep. Journal of Agricultural Science, Cambridge, v.105, n.2, p.271277, Apr. 1985.

SOLOMON, R.; YOSEF, E.; MIRON, J.; DROR, Y; BENGHEDALIA, D. Absorption of macro- and microelements by sheep from barley and barley plus sulphur dioxide-treated straw rations. Small Ruminant Research, Amsterdam, v.6, n.1/2, p.5562, Oct. 1991

TERNOUTH, J.H.; DAVIES, H.M.S.; MILLION, J.T.B.; SIMPSON-MORGAN, M.W.; SANDS, N.E. Phosphorus metabolism in ruminants. I. Techniques for phosphorus depletion. Australian Journal of Agricultural Research, Melbourne, v.36, n.4, p.637-645, June 1985.

THOMPSON JUNIOR, W.R. Phosphorus in animal nutrition. In: PHOSPHORUS for agriculture: a situation analysis. Atlanta: Potash/Phosphate Institute, 1978. p.126-158.

UNANIAN, M.M.; SILVA, A.E.D.F. Valores bioquímicos no soro sangüíneo de cabras, relacionados ao estado fisiológico e raça no nordeste semi-árido. II. Cálcio, fósforo e magnésio. Pesquisa Agropecuária Brasileira, Brasília, v.20, n.10, p.1223-1228, out. 1985.

VITTI, D.M.S.S. Avaliação da disponibilidade biológica do fósforo do fosfato bicálcico, Patos de Minas, Tapira e finos de Tapira para ovinos pela técnica de diluição isotópica. São Paulo: Instituto de Pesquisas Energéticas e Nucleares, 1989. 72p. Tese de Doutorado.

VITTI, D.M.S.S.; ABDALA, A.L.; SILVA FILHO, J.C da. Avaliação da disponibilidade biológica do fósforo do fosfato bicálcico e de fosfatos de rocha para ovinos com o uso do radiofósforo $\left({ }^{32} \mathrm{P}\right)$ como traçador. Pesquisa Agropecuária Brasileira, Brasília, v.26, n.8, p.1113-1118, ago. 1991.

VITTI, D.M.S.S.; ABDALA, A.L.; SILVA FILHO, J.C. da; AMBROSANO, E.J. Métodos para o diagnóstico da deficiência de fósforo em ruminantes. Pesquisa Agropecuária Brasileira, Brasília, v.23, n.6, p.645-651, jun. 1988 . 